\title{
Applying of PAW for Standarization of CsI(TI) Light Output
}

\author{
Joanna Borgensztajn \\ Institute of Physics, University of Zielona Góra \\ ul. Prof. Szafrana $4 a$ \\ 65-516 Zielona Góra, Poland \\ e-mail: J.Borgensztajn@proton.if.uz.zgora.pl
}

(Received: 23 March 2010; accepted 12 July 2010; published online: 27 September 2010)

\begin{abstract}
The basis of a new method of automatic particle identification for CsI(Tl) scintillators is described and discussed. A useful tool for this purpose is PAW (Physics Analysis Workstation). PAW is an interactive and object-oriented utility for visualizing data, statistical analysis and some other tasks, which combines different CERN packages. A special procedure for CsI(Tl) data analysis, running under PAW in a batch mode, has been written and tested. The recent paper presents the main idea of the method and results obtained for simulated data. As the first step of the test, a relatively simple case of data has been examined. Obtained results indicate that for more complicated cases the procedure should work effectively as well.
\end{abstract}

Key words: $\operatorname{CsI}(\mathrm{Tl})$ scintillators, Physics Analysis Workstation, pulse shape

\section{INTRODUCTION}

CsI(Tl) scintillators are often used in nuclear and high energy physics as charged particles detectors. Passing through the material, a particle causes flashes of light called scintillations. A detailed and comprehensible for a non-specialist description of the scintillation process in a broad range of materials may be found in [1].

Optical properties of $\mathrm{CsI}(\mathrm{Tl})$ allow simultaneous measurement of different types of the particles and quite good discrimination between them. The last possibility is related to the fact that the duration of the light pulse emitted by some materials is determined by two time constants. The first one corresponds to the so called fast component of emitted light and varies very strongly with particle mass, charge and energy. The second corresponds to the so-called slow component and is regarded as independent of a particle type.

By recording the pulse shape and applying the Pulse Shape Discrimination Method [2], it is possible to find for each event two digital variables proportional to the fast and slow component respectively. Data analysis is usually done by displaying these variables on two-dimensional fast-slow plot (see examples in [2-4]) and by putting graphical cuts or masks separating isotopic branches.

The idea of this method is very simple but it should be noted that for two or more identically fabricated detectors we never obtain identical fast-slow plots. Moreover, plots obtained for one detector vary with temperature. Ageing effects of scintillators are also present so the procedure of putting masks should be repeated many times, according to the current conditions. In the case of devices consisting of several hundred detectors as CHIMERA [2] or INDRA [4] the method is a very time consuming process.

To overcome this difficulty, a modification of the commonly used method is proposed. The modification is based on the concept that all fast-slow plots obtained for one or more detectors could be standardized to one pattern.

\section{DATA FILES PREPARATION}

Isotopic branches on fast-slow plots can be described by empirical formula:

$$
s=(a(A, Z) f+b)^{n},
$$


where $f, s$ represent fast and slow component respectively, $a(A, Z)$ is a parameter dependent on mass and charge of the particle, $b, n$ are parameters dependent on individual properties of the detector (J. Borgensztajn, personal communication, IWASOM'08, Gdańsk, Poland).

Using the formula (1) branches for ${ }^{1} \mathrm{H},{ }^{2} \mathrm{H},{ }^{3} \mathrm{H}(Z=1)$, ${ }^{3} \mathrm{He},{ }^{4} \mathrm{He},{ }^{6} \mathrm{He}(Z=2)$ and ${ }^{6} \mathrm{Li},{ }^{7} \mathrm{Li},{ }^{8} \mathrm{Li}(Z=3)$ have been simulated. In both cases the same set of $a(A, Z)$ parameters has been used. All parameters have been chosen arbitrarily to reproduce experimental plots roughly, so there is no necessity to go into details of the simulation.

Simulated data had been written as Ntuple files, which means the main type of files used by PAW. Then the data were displayed in $f-s$ coordinates. Examples of plots are shown in Fig. 1.

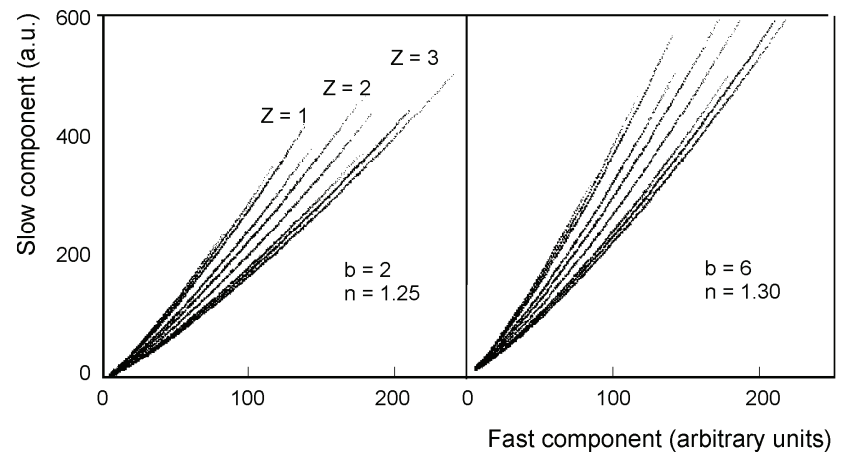

Fig. 1. Simulated fast-slow plots. The simulation very well reproduces differences observed for experimental data

\section{STANDARDIZATION OF FAST-SLOW PLOTS}

To simplify the procedure of putting masks on the fastslow plots, it would be useful to find a coordinate system in which isotopic branches are straight lines. We can obtain that result by displaying the data in $f-s_{1}$ coordinates (not shown), where

$$
s_{1}=\sqrt[n]{s}-b
$$

In the new coordinates we can define for each event $\Theta$-angle measured from $s_{1}$-axis to isotopic branches. Both $\Theta$ like its any trigonometric function can be considered as a parameter explicitly identifying a type of particle, $f$ is related to particle energy.

The main idea of isotopes identification in $f-\sin \Theta$ coordinates is shown in Fig. 2. In this case, to identify an isotope it is enough to determine the lower and upper range of any branch $\left(r_{l}\right.$ and $\left.r_{u}\right)$ and to put on the data logical condition

$$
r_{l}<\sin \Theta<r_{u} .
$$

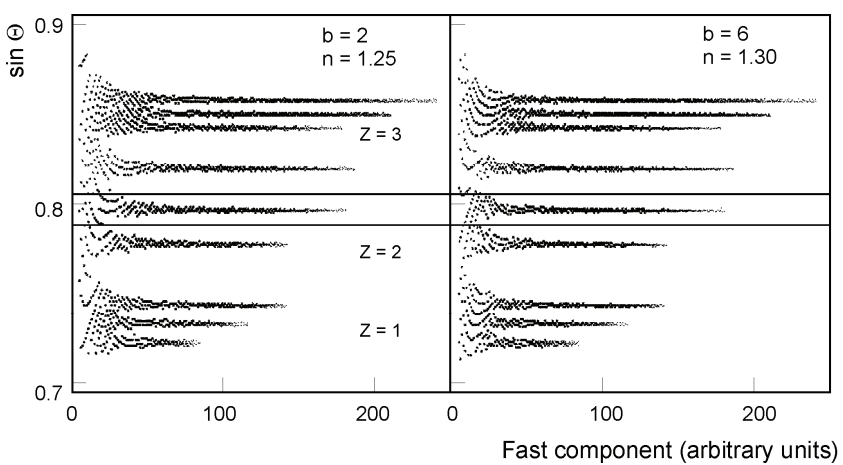

Fig. 2. The proposed particle identification method: an isotope (in this case ${ }^{4} \mathrm{He}$ ) can be identified in $f-\sin \Theta$ coordinates by logical condition $r_{l}<\sin \Theta<r_{u}$

Data distribution of ${ }^{4} \mathrm{He}$ along $f$-axis is presented in Fig. 3. The black line represents a given distribution compared with the distribution of identified events (hatched area). From Fig. 2 it is clear that most of the points which fulfill the relation (3) may be unambigously identified. Only for a small fraction of points, particularly those with small values of the fast component, the identification is ambiguous. That fraction is excluded from the hatched area.

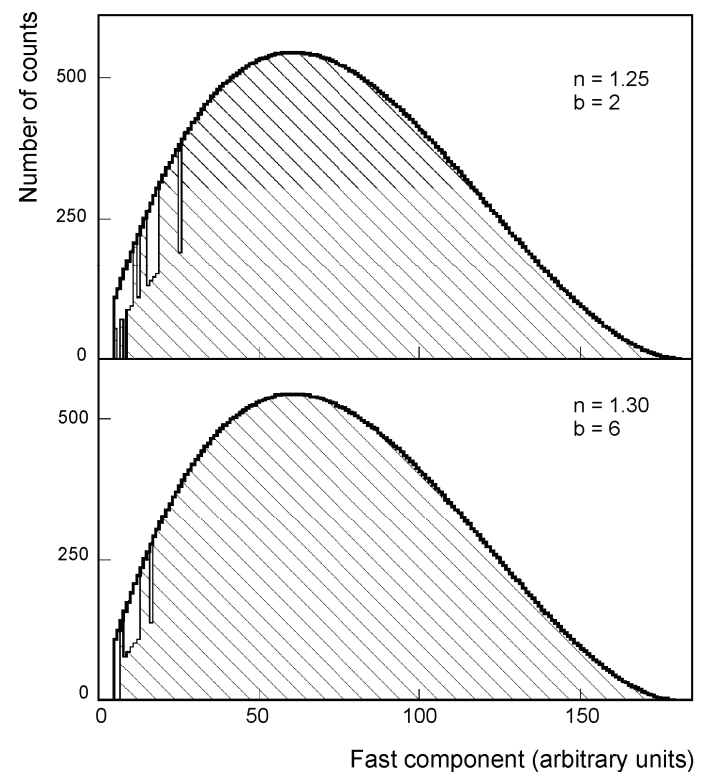

Fig. 3. Comparison between given distribution of ${ }^{4} \mathrm{He}$ along $f$-axis (black line) and the distribution of identified events (hatched area) 


\section{CONCLUSIONS AND PERSPECTIVES}

The proposed procedure for particles identification is much easier to apply and less time consuming than traditional methods, especially under PAW. Simulated data have been standardized to one pattern by using $f-\sin \Theta$ coordinates (Fig. 2). As it is shown in Fig. 3, the procedure loses not many events, which is practically impossible in the case of putting graphical cuts.

For experimental data we can expect a more complicated situation: $f-\sin \Theta$ plot obtained for one scintillator may be shifted or/and scaled in comparison with plots obtained for the other ones. In this case, it is possible to modify the procedure and to use some calibration para- meters. A more detailed description of the latter idea will be published soon.

\section{References}

[1] J.B. Birks, The theory and Practise of Scintillation Counting. Pergamon Press, Oxford 1964.

[2] M. Alderighi et al., Particle identifcation method in the CsI(Tl) scintillators used for the CHIMERA $4 \pi$ detector. Nucl. Instr. and Meth. A489, 257-265 (2002).

[3] S. Aiello et al., Light response and particle identifcation with large CsI(Tl) crystals coupled to photodiodes. Nucl. Instr. and Meth. A369, 50-54 (1996).

[4] J. Pouthas et al., INDRA, a $4 \pi$ charged product detection array at GANIL. Nucl. Instr. and Meth. A357, 418-442 (1995).

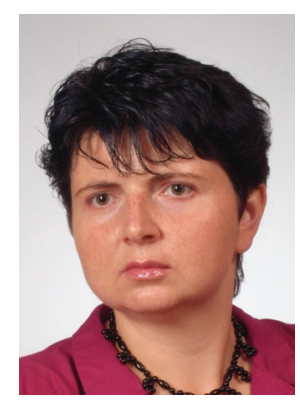

Dr. JoAnNa BorgensztaJn deals mainly with nuclear physics. The latest area of her interest has been data analysis of fast-slow matrices obtained for $\mathrm{CsI}(\mathrm{Tl})$ scintillators by CHIMERA collaboration. Recently she has been working at the Institute of Physics of the University of Zielona Góra, Poland. 